\title{
LA OTRA REFORMA: EL "NO SEXISMO" COMO CLAVE CULTURAL DEL CAMBIO EN EL SISTEMA EDUCACIONAL
}

Maria Elena Acuña Moenne y Sonia Montecino Aguirre 


\section{MARÍA ELENA ACUÑA MOENNE}

Antropóloga social de la Universidad de Chile; Doctora en Estudios Americanos, mención Pensamiento y Cultura, Usach. Académica del Departamento de Antropología de la Universidad de Chile.

\section{SONIA MONTECINO AGUIRRE}

Antropóloga de la Universidad de Chile; Doctora en Antropología de la Universidad de Leiden, Holanda. Profesora Titular del Departamento de Antropología de la Universidad de Chile. 


\section{LA OTRA REFORMA: EL “NO SEXISMO” COMO CLAVE CULTURAL DEL CAMBIO EN EL SISTEMA EDUCACIONAL}

\section{PRESENTACIÓN}

El propósito de este artículo es proponer algunas claves para observar la relación entre el género y el sistema educativo, visto este último a través de las dinámicas que suceden en los establecimientos donde se vehiculizan los procesos de enseñanzaaprendizaje. La relación de las mujeres con el sistema educativo es larga y antigua y nos habla de exclusiones e incorporaciones, procesos con momentos de contracción y expansión, pero que sin duda alguna han significado avances en los derechos de las mujeres. En pleno 2014 nos encontramos en un momento de expansión del tema toda vez que, socialmente, se está comenzando a esbozar la preocupación por la calidad del habitar de hombres y mujeres en los espacios tanto escolares como de educación superior. Esto ha hecho emerger nuevas temáticas y demandas en torno a las formas sexistas y heteronormativas que adquiere la educación en general, que sanciona cuerpos, conocimientos e identidades que no se ajustan a las reglas que impone el sistema, imposiciones que se realizan, como sabemos, a veces de manera sutil y otras de modo más abierto.

Primero haremos un recorrido por la relación género y educación desde un punto de vista histórico institucional y luego abordaremos algunas propuestas sobre los elementos necesarios para avanzar en la construcción de un sistema escolar no sexista.

\section{GÉNERO Y EDUCACIÓN: UN TRÁNSITO NUNCA FINALIZADO}

Desde muy temprano en el siglo XIX, en las estrategias de desarrollo de Chile, se estableció la importancia de la educación para el "avance" del país, así como para la construcción de formas identitarias nacionales de carácter masivo. La escuela se instaló como espacio de enseñanza, pero adquirió dimensiones simbólicas de gran alcance, transformándose en un símbolo de la patria, la civilización, la letra, la norma y la ley. En 1827 las condiciones sociales y políticas permitieron la creación de escuelas para mujeres (tanto laicas como conventuales), así como también las escuelas para preceptores (las famosas escuelas normales). El año 1860 se promulgó la Ley de Instrucción Primaria, que definió que el Estado sería el principal y único 
actor en la administración y definición del sistema escolar ${ }^{15}$, a la vez de ser el principal empleador de este sector, razones por las que se acuñó la idea de "Estado Docente" (Labarca 1939).

Los primeros años de implementación de esta ley destacaron por la creación de un ideal social que puso en la educación las esperanzas sobre el progreso, la movilidad y la inclusión social, que formaban parte de las demandas de una cada vez más organizada clase obrera, sobre todo si se considera que en ese momento la sociedad chilena estaba en un proceso de transformación y de resignificación de los principios culturales propios de la Colonia Española, -tales como las ideologías sobre los indígenas, la raza y las mujeres-. Hacia la segunda mitad del siglo XIX, grupos de mujeres comenzaron a demandar por el acceso a la educación, a la capacitación y al ejercicio profesional (Valdivieso 1995; Lavrin 2005 ), demandas que se vieron contestadas el año 1877 cuando el Ministro de Instrucción Pública del Gobierno del Presidente Aníbal Pinto, establece a través de un decreto -conocido como el decreto Amunátegui- que

"las mujeres deben ser admitidas a rendir exámenes válidos para obtener titulos profesionales, con tal que se sometan para ello, a las mismas disposiciones a que están sujetos los hombres" (Decreto Amunátegui, 1877).

Como hoy día es sabido, una ley no implica un cambio cultural, y en el caso de las mujeres es casi una regla. La posibilidad de las mujeres de ingresar a la Universidad de Chile, la única existente en aquel entonces, no fue fácil. En nuestro país se ha prestado poca importancia a la reflexión sobre cómo los regímenes de género se expresan y afectan la presencia de mujeres y hombres en el sistema educativo, y muchas veces ponemos la mirada en la existencia de un momento inicial, más bien fundacional, un dispositivo que imprimió fuerza a procesos de cambio cultural, pero cuya capacidad para generar una transformación cultural es evidentemente baja, en este sentido no produce ni articula lo que autoras preocupadas de las transformaciones culturales de género denominan una brecha de género ${ }^{16}$ (Ortner 1990). Aun pasados los primeros 15 años del siglo, y a más de cien años

15 El Estado, a través del ministerio que cubría el área de educación -cartera que ha tenido varios nombres- tuvo la capacidad de fijar los planes y programas y administrar el sistema a través de mecanismos centralizados, hasta la década de 1980.

16 Alude a coyunturas históricas que facilitan el cambio en los regímenes de género aun cuando estos no formen parte de los proyectos políticos en disputa o tensión. 
seguimos valorando la gesta política de aquellas mujeres que fueron las primeras en matricularse, en graduarse y tal vez, en ejercer una profesión.

El proceso de incorporación de las mujeres como estudiantes y luego en tanto trabajadoras como docentes, directivas e investigadoras en las escuelas, liceos y universidades a lo largo del siglo XX, forma parte de un largo recorrido de demandas en busca del ejercicio de la ciudadanía sustantiva para las mujeres; que avance en el reconocimiento de la participación que tenemos en la reproducción social. Las demandas que grupos de mujeres realizaban en torno a su acceso a la educación se enmarca dentro de procesos relacionados con el acceso a la ciudadanía que comenzaron muy tempranamente en el siglo XIX; la modificación del régimen de género en orden a permitir el desarrollo de otras prácticas identitarias y de posicionamiento social que avanzaron muy ligadas a las demandas por la participación política. La articulación de demandas educativas fue una etapa relevante en el largo proceso político de construcción de nuevas formas de ciudadanía y participación para las mujeres (Valcarcel 2001); en este camino el acceso a la educación superior es un gran logro político y cultural.

Las narrativas sobre el ingreso de las mujeres a la universidad en nuestro país adoptan una forma de saga donde las protagonistas son las pioneras, las primeras mujeres en ingresar a estos espacios y titularse. No ahondaremos en esta información debido a que existen numerosos recuentos biográficos sobre ellas, sin embargo, queremos destacar dos cosas al respecto. La primera se refiere a la necesidad permanente de recordar esta etapa fundacional y establecer a través de ella la cartografía del recorrido femenino por el acceso a una ciudadanía que pueda ser construida y ejercida de múltiples maneras, donde educarse y la educación resultan fundamentales. Cada una de las mujeres que hoy día conocemos como las primeras médicos, ingenieras, abogadas (entre otras profesiones) marca un tipo de problemática en este tránsito y se transforma en un nudo de memoria que permite recrear permanentemente las tensiones de género que articula el sistema educativo. Por un fenómeno más bien administrativo tendemos en la actualidad a seccionar la experiencia educativa en los niveles educativos, pero las personas viven su paso por el sistema más bien como un continuo, donde las discriminaciones vividas se consolidan como una experiencia (Guerra, 2004).

El segundo hecho se refiere a la duración del proceso de incorporación de las mujeres en las universidades chilenas y el marco más general en el que este se produce y se concreta. Esta discusión aborda la relación entre la educación y el proyecto de país. Cuando se dicta el decreto Amunátegui no existía en Chile ningún tipo de dispositivo legal que garantizara la instrucción de la población. Esto recién pasaría en la década de 1920, cuando se estableció la obligatoriedad de la 
instrucción primaria, que hoy conocemos como enseñanza básica ${ }^{17}$; además esta enseñanza estaría en manos del Estado chileno.

En esa época se estimó que el Estado tenía la suficiente capacidad para educar a todos los habitantes del país, pero producto de las redefiniciones que empezaban a operar en las estrategias de desarrollo el énfasis escolarizador estuvo puesto en las zonas urbanas y en la parcialidad masculina de la población, en concordancia con la articulación de un proyecto industrializador ${ }^{18}$. Recién hacia la década de 1950 la Ley de Instrucción Primaria Obligatoria se comenzó a aplicar en las zonas rurales; no obstante, este evidente avance en términos de la democratización social no fue un indicador de aumento en la equidad de género toda vez que la ampliación de la matrícula escolar femenina fue más lenta que la masculina. (Soto 2000).

En la década de 1960, con la ampliación de la ciudadanía, la definición del sistema educacional fue un campo político de tensión entre los sectores conservadores, los movimientos obreros y los partidos políticos de izquierda, estableciéndose propuestas para su transformación en los planes de gobierno en las décadas de 1960 y 1970. Sin duda alguna, el golpe militar del año 1973 frenó, desarticuló y hasta "satanizó", algunas de estas propuestas como, por ejemplo, la Escuela Nacional Unificada (ENU). Las autoridades dictatoriales esperaron algunos años antes de intervenir directamente el sistema escolar de manera articulada y organizada, no obstante la represión a los/as docentes y estudiantes y la censura de contenidos curriculares y materiales educativos que operó desde los primeros días posteriores al golpe de Estado.

La intervención militar del sistema educativo se plasmaría en la década de 1980 debido a la confluencia de tres factores: la crisis económica mundial, el carácter ideológico antipopular de la dictadura militar y el fortalecimiento a nivel mundial de las políticas neoliberales que facilitarían una modificación sustancial del sistema educacional chileno desarticulando el principio que la había regido hasta ese entonces y la educación dejó de ser pública y, por lo tanto, el acceso y cobertura universal para todos los integrantes de la sociedad chilena se puso en jaque.

Parte de las reformas neoliberales se orientaron a modificar el rol del Estado a través de la reducción de su presupuesto y su personal, lo que para el caso de las políticas educativas significó el fin del Estado Docente, especialmente producto de las reformas conocidas popularmente como municipalización de la educación. Estas permitieron redistribuir recursos a través de dos medidas; la primera de

17 Estableció que la educación primaria debía ser gratuita, laica y obligatoria.

18 En concordancia con el proyecto industrializador se fundó en 1947 la Universidad Técnica del Estado y se crearon las facultades de Economía y Comercio de la Universidad de Chile. 
ellas se relaciona con la incorporación de sectores privados en la administración y definición del proceso educativo por medio de la implementación de un sistema de subvenciones que todavía permite al Estado otorgar recursos a los establecimientos educativos privados en el mismo nivel que a los establecimientos públicos, y se termina con el funcionamiento centralizado del sistema que estaba, hasta ese momento, en manos de un único actor, el Ministerio de Educación, que fijaba los planes y programas para el conjunto de los establecimientos del país (Soto 2000, Carvajal 2004).

La segunda se debe a que, en concordancia con las medidas de ajuste estructural que indicaban la disminución del aparato estatal, se promulgan los Decretos con Fuerza de Ley No 3063 de junio 1980 y el No 3476 de septiembre de 1980, que reglamentan el traspaso de los establecimientos educacionales de enseñanza básica y media y su personal, desde el Ministerio de Educación a las Municipalidades. Hasta ese momento el Ministerio de la cartera de educación dictaba las normas y contenidos curriculares; designaba a los profesores y directivos; construía y reparaba la infraestructura educacional; asignaba y pagaba los costos y remuneraciones y adquiría y distribuía los insumos escolares. El porcentaje de establecimientos educacionales públicos que el Ministerio de Educación tutelaba hasta ese momento ascendía al 80\% del total de escuelas del país, llegando a administrar el 90\% del presupuesto del sistema, temas ampliamente debatidos en la actualidad.

En términos de los lineamientos de la geopolítica del gobierno militar, esta reforma se enmarcaba dentro de un proceso más general y amplio, denominado descentralización, que implicó transformaciones en la administración general del país, a la vez que se daba cumplimiento a las normativas del Banco Mundial en materia educativa que establecían que la privatización de la educación así como su administración descentralizada eran las condiciones que permitirían la elevación de los estándares de calidad educativa (Reimers 1990).

Con el advenimiento de la democracia y de los gobiernos de la Concertación de Partidos por la Democracia (1990 en adelante), se planteó un cambio radical a los contenidos de la educación, pero manteniendo el orden estructural de colegios municipalizados, particulares subvencionados y particulares. La transformación propuesta por el Ministerio y avalada por las políticas económicas internacionales de ciertas instituciones (como el Banco Mundial), propugnó un cambio en las formas de enseñanza -por tanto un cambio en los métodos pedagógicos- en donde se privilegiará el "constructivismo" (es decir que los/as alumnos/as realicen su propio aprendizaje a partir de elementos que pertenecen a su realidad). Asimismo se propuso una reforma en los contenidos de los programas y una nueva designación de sectores y subsectores que reagruparon a las asignaturas y su denominación: Lengua Castellana y Comunicación; Historia y Ciencias Sociales; aumento del presupuesto y 
focalización de intervenciones de emergencia en sectores cuyos resultados escolares eran preocupantes.

El diagnóstico crítico de la situación de la educación en Chile pasó a formar parte del discurso político de la transición a la democracia hacia fines de la década de $1980^{19}$ y la necesidad de su reforma se instaló como una prioridad en los programas de gobierno a partir de la década de 1990, que a partir de entonces comenzaron a desarrollar diversas acciones para producir una transformación del sistema educativo nacional orientadas a intervenir en el sistema de financiamiento y en el proceso de enseñanza aprendizaje. Sin embargo, no hubo una preocupación ni por el racismo ni por el sexismo que el sistema educativo reproduce; entendiendo que las exclusiones no sólo son por las dificultades de acceso, sino por la invisibilidad de sujetos históricos y personas con experiencias que se dislocan de las lógicas heteronormativas que el sistema educativo, como reproductor de la cultura oficial, promueve.

Durante la década de 1990, los procesos de la reforma educacional posibilitaron una discusión muy parcial sobre los temas de equidad de género. Por un lado, la equidad de género se introdujo como lineamiento a partir de 1990 con el Plan de Igualdad de Oportunidades para Hombres y Mujeres que estableció la necesidad de investigar sobre el sistema educacional para poder:

"entender por qué-pese a que la participación femenina es numéricamente paritaria en las matriculas de enseñanza básica y secundaria y al mayor rendimiento escolar de las niñas-existe una segmentación sexual muy pronunciada en la elección de carreras y especialidades, que contribuye a la discriminación de las mujeres en sus posibilidades económicas y profesionales futuras, (de ahí la necesidad de establecer) lineamientos y acciones de las normas que favorezcan la generación de oportunidades igualitarias para las mujeres" (Sernam 1994: 25).

Pregunta que seguimos sin responder, no porque esté mal formulada, sino porque nuestra mirada no ha sido la adecuada. Aun cuando se avanzó en algunas nociones sobre la educación no sexista, a la larga el centro del debate y las acciones implementadas fue, casi exclusivamente, la educación sexual, como la Política

19 Según Arellano (2001) los elementos diagnósticos sobre el estado de la educación en Chile fueron:

- Cobertura prácticamente universal en la Educación Primaria, y cercana al 80\% en la Secundaria; caída del gasto público en educación y a la expansión en la matrícula del nivel medio, baja calidad de aprendizaje, bajas remuneraciones docentes; doble dependencia administrativa de los establecimientos de los municipios para aspectos administrativos, y del Ministerio de Educación respecto a currículum, pedagogía y evaluación. 
de Educación en Sexualidad (1993) y, la Constitución de la Comisión Intersectorial para la Prevención del Embarazo Adolescente de donde emanó el diseño de las JOCAS - Jornadas de Conversación sobre Afectividad y Sexualidad- un programa que en su momento fue bastante controversial y que desató críticas de sectores conservadores por explicar sobre el uso de preservativos. El levantamiento de información estadística a nivel nacional sobre el impacto del embarazo adolescente en la Educación Básica y Media, que concluyó con la redacción de la circular 247 para la no-discriminación de las alumnas embarazadas el año 2000.

Junto con las acciones en el ámbito de la educación sexual, se revisaron los contenidos de los materiales educativos y promovido una supuesta equidad en la representaciones de hombres y mujeres en los materiales educativos; esfuerzos que, sin embargo, no tuvieron continuidad en lo que va de corrido del XXI.

Las demandas por educación no sexista, cada vez más visibles a partir del año 2011, tienen esta vez otro foco. Emergen de la demanda de las propias estudiantes. Existen numerosos colectivos y agrupaciones de mujeres jóvenes y hombres feministas que han venido a reinstalar el tema y a resignificar las demandas, incluyendo esta vez las discusiones sobre los derechos sexuales y reproductivos, los derechos identitarios y el respeto por las personas LGTB ${ }^{20}$. Temas y diferencias que el sistema escolar no admite. Solo recordemos que en las condiciones actuales de funcionamiento, muchas escuelas no permiten ni siquiera a los niños inquietos.

$\mathrm{Al}$ igual que la vida cotidiana de las primeras mujeres que ingresaron a la Universidad a fines del siglo XIX, las vidas de los actuales niñas, niños y adolescentes se ve fuertemente entretejida con las ideologías de género que construyen y representan a las mujeres como inferiores o incapaces en muchas áreas del conocimiento; ideologías muchas veces naturalizadas, por lo que su desmontaje es difícil y complicado. El recuerdo de esta etapa fundacional de la relación educacióngénero-derechos parece ser necesaria como un impulso que reinstala de manera permanente los efectos discriminadores sobre las mujeres y otros grupos periféricos respecto de los núcleos de poder del sistema educativo y de la sociedad en general.

Como ampliación de este impulso y a modo de gesto también lo ponemos en la escena de esta discusión, para plantear la necesidad de que la actual discusión sobre la reforma educativa del año 2014 avance en el abordaje de las dimensiones culturales del sistema educativo, su relación con la articulación de discriminaciones que se reproducen de manera histórica y un reconocimiento a las agrupaciones de mujeres que hoy día plantean la necesidad de transformar precisamente las matrices

20 Sigla usada para referirse a lesbianas, gays, bisexuales y transexuales. 
culturales sexistas y heteronormativas ${ }^{21}$ que se promueven en la sociedad y cuyo aprendizaje es parte de lo que se ha llamado el currículum oculto de género (Acuña 2006). Puede que sus prácticas y expresiones cambien, pero también es cierto que las movilizaciones del 2011 encontraron, en las teoría feministas, queer y de los feminismos postcoloniales, conceptos muy precisos para expresar el malestar por los modelos de género-sexualidad, de clase y étnico que los procesos de enseñanza y aprendizaje promueven.

Así como en la sociedad en general, en el sistema educativo en particular, las relaciones de género, étnicas y de clase son factores estructurantes de los grados y las características de la discriminación hacia las mujeres y los hombres que se ubican en posiciones marcadas.

\section{REPENSAR LA ESCUELA}

La demanda que se expresa hoy día respecto de una educación no sexista no sólo nos parece válida, sino que legítima y necesaria para que escuelas, liceos y universidades se forjen como espacios con contribuciones reales a la democracia y la equidad en el país. Consideramos en extremo destacable el movimiento silencioso que se gesta en torno a este tema en la actualidad.

Del mismo modo, compartimos las inquietudes respecto de qué es o cómo sería una escuela no sexista. Preguntas difíciles de responder, pues no existe una única posición al respecto.

Primero porque el modo en que el género es definido y usado en muchos trabajos y estudios sobre educación presenta una amplia variedad de definiciones y aproximaciones (Thompson 2003). En nuestro caso particular, observamos las relaciones de género a través de las posibilidades de las teorías antropológicas y, por lo tanto, nuestra definición sostiene que el género es una construcción cultural, y como tal se transforma históricamente. La cultura, siguiendo a Geertz (1995) es un conjunto de tramas de significación urdidas por seres humanos en sus relaciones y contextos cotidianos. La cultura es, por tanto, un esquema históricamente transmitido de significaciones representadas en símbolos, un sistema de concepciones heredadas y expresadas en formas simbólicas por medio de las cuales los seres humanos se comunican, perpetuán y desarrollan su conocimiento y sus actitudes frente a la vida. El género, por lo tanto, es el conjunto específico de tramas de significación respecto de la lectura cultural, primero, sobre las diferencias corporales entre hombres y mujeres; luego de las construcciones identitarias, roles y posiciones sociales para los

21 Modelo de género que implica la existencia de dos sexos, dos y la heterosexualidad. 
sujetos en función de su clasificación inicial. En nuestra sociedad y nuestra cultura estas posiciones y definiciones se establecen hegemónicamente como binarios, a cada cuerpo una identidad posible. Pero sabemos que esto no es una regla ni una constante universal, y deberíamos decir real. Es lo que se ha denominado como la hegemonía de los puntos de vista heteronormativos. Finalmente, cuerpos e identidades se despliegan como un abanico de posibilidades.

Pero volvamos a nuestra pregunta utópica.

Una escuela no sexista debería ser aquella que, antes que nada, reconoce que los procesos de enseñanza-aprendizaje se interdigitan con las construcciones de género. Dicho de otro modo, debe partir por reconocer las formas en que los regímenes de género se expresan en todas las funciones y tareas educativas y las formas en que afectan la presencia de mujeres y hombres, prestando atención de que esto no solo refiere a los(as) estudiantes, sino también a profesores(as) y administrativos.

En segundo lugar, proponemos como necesaria una mayor conciencia sobre los procesos biológicos, que también son significados culturalmente y que se orientan a considerar con mayor claridad que el cuerpo -en tanto experiencia cultural- es un espacio de derechos. El cuidado y conocimiento del cuerpo, la promoción de un lenguaje no discriminador sobre el aparato reproductor de hombres y mujeres; sobre procesos fisiológicos como la menstruación y el embarazo; pero también un abordaje de situaciones que hoy día nos desafían socialmente, como las problemáticas de las personas transgénero. Es necesario hacer un esfuerzo político por dejar todo tipo de prácticas que intenten la normalización de todo lo que escapa al patrón heteronormativo.

A modo de ejemplo, hace muchos años un colega que realizaba etnografías de aula en un liceo de la comuna de Nuñoa, en el marco de un proyecto Fondecyt que compartimos, nos comentó que estaba muy impactado al haber escuchado a un profesor decir en clases de biología: "ojalá las mujeres fueran como las gallinas, porque cacarearían cuando están disponibles". Sólo hay que preguntarse cuántos de estos microprocesos de discriminación y poder se viven de manera habitual en los establecimientos educacionales.

En tercer lugar, una revisión de las perspectivas del conocimiento que se transmiten. El libro de Celia Amorós Hacia una crítica de la razón patriarcal, publicado hace varias décadas, propone una revisión de las tramas ideológicas del conocimiento occidental, pues claro que sí, el conocimiento no es neutro ni objetivo como algunos sectores se esfuerzan en hacernos creer. Como se plantea en dicho texto, el sexismo no sólo afecta la razón teórica, sino también la razón práctica. Amorós (1991) plantea su uso del término ideología en el sentido marxista de una percepción distorsionada de la realidad en función de unos intereses de clase, concepción que puede ser ampliada a cualquier deformación específica de la visión y valoración de los hechos 
condicionados por las necesidades de un determinado sistema de dominación, y el hecho de que la mitad de la especie humana se encuentra en situación de enajenación y marginación necesariamente tiene consecuencias gnoseológicas distorsionantes en los procesos de producción y legitimación de conocimientos.

En la actualidad, la estructuración del campo de conocimiento produce varias exclusiones: primero no considera como centrales los conocimientos producidos por mujeres, aun cuando estos sean creados en laboratorios regidos por el occidental método científico; en segundo lugar, la historia de las mujeres y otros grupos subalternos no es parte de las narrativas escolares. Solo a modo de ejemplo, la preocupación sobre la participación de las mujeres en la ciencia, que es baja y complicada a nivel mundial, cómo puede ser revertida si las niñas y adolescentes no son incentivadas con las trayectorias de mujeres que les muestren que es posible, a pesar de las torsiones que muchas veces deben realizar con sus vidas. Asimismo, es necesario integrar en el proceso escolar conocimientos generados al amparo y cobijo de actividades propiamente femeninas en cada sociedad. ¿Qué conocimientos, asociaciones y operaciones lógicas y matemáticas entraña la producción de un textil, de un bordado o la preparación de una rica cazuela?

Nosotras mismas no tenemos la respuesta, pero sí la claridad en plantear que en la actualidad el sistema escolar excluye y margina muchos tipos de conocimientos. Su alterofobia también se expresa en la denigración de no oficial y de los conocimientos específicos de los pueblos nativos.

La folclorización es el dispositivo más usado, fijando muchas prácticas como ahistóricas o en proceso de desaparición, dignas de conocer por un ejemplo de lo "extraño". Los llamados "procesos tradicionales" de producción de conocimientos y desarrollos tecnológicos no alcanzan el estatus científico de "conocimiento" propiamente tales, por lo tanto, se deja a las poblaciones productoras de los mismos en una situación de precariedad y vulnerabilidad (Hobart 1993).

En tercer lugar, el sistema educativo debe estar preparado para el desarrollo e implementación de una didáctica no sexista que encare las actuales tendencias, preocupantes, de rearticulación neomachista. Nos referimos a todas aquellas prácticas que bajo una aparente postura de igualdad -por tratarse del discurso políticamente correcto de la sociedad- encubren violencias simbólicas, sesgos, discriminaciones y desvalorizaciones de lo femenino y de lo "diferente". Son las conductas que se deslizan en pasillos, a la hora de colocar una nota, al momento de decidir quién ocupa un sitio, quién debe ser premiado(a), incluso quién y cómo debe ser escuchado en una sala de clases. El neomachismo es una fuerza que se gesta a contracorriente de los avances del feminismo, de los grupos que reivindican sus diferencias sexuales y que con un discurso progresista ejecuta prácticas de marginación y subordinación. 
Nuevas formas de violencia hacia las mujeres, gays, lesbianas y transgéneros emergen en el contexto neoliberal global. La mercantilización de las experiencias de vida diferentes, las exclusiones basadas en el cuerpo, las prácticas de aniquilamiento de las diferencias a través de la agresión y desaparición del cuerpo, las mutilaciones, etc. No estamos diciendo que esto sucede en el sistema escolar, pero tampoco este está respondiendo al proceso de transformación cultural, marcado por la fobia a todo lo "diferente".

Una de las autoras de este artículo tiene un hijo y una hija en edad preescolar. El año pasado, cuando su hijo cursaba prekínder, el niño le contó que habían escuchado el cuento Caperucita y el lobo y que al final del relato habían jugado al cuento cuya centralidad era que los hombres eran los lobos que se comían a las mujeres. Sin duda alguna tuvimos acceso a un momento poco conocido por la teoría, un momento inicial de construcción de representaciones de género y violencia. Pero para desarmarla, se requiere preparación teórica especializada que hoy días los(as) docentes y los mismos padres no poseen con claridad.

La didáctica no sexista debe ser consciente de las diferencias, pero también de la igualdad. La igualdad no es la igualación, sino la claridad ética de entender que los niños y las niñas, las adolescentes y los adolescentes son sujetos de derechos y especialmente de los derechos sobre su posición de género en la sociedad.

\section{REFERENCIAS}

Amorós, C. (2001). Hacia una crítica de la razón patriarcal. Madrid: Anthropos editorial. Carvajal, R. (2004). Fundamentos de la calidad de la educación en Chile: de lo macro a lo micro. Universidad de Chile, Facultad de Ciencias Económicas y Administrativas. Memoria para Optar al Título de Ingeniero Comercial.

Geertz, C. (1995). La interpretación de las culturas. Barcelona: Gedisa.

Guerra, M.A (2004). Arqueología de los sentimientos en la organización escolar. En Tendencias Pedagógicas, pp.45-69.

Labarca, A. (1939). Historia de la enseñanza en Chile. Santiago: Imprenta Universitaria. Lavrin, M. (2005). Mujeres, feminismos y cambio social en Argentina, Chile y Uruguay 18901940. Santiago: Centro de Investigaciones Diego Barros Arana.

Ortner, Sherry (1990). "Gender Hegemonies”. En: Cultural Critique, Winter 19891990, pp. 35- 80.

Reimers, F. (1990) The impact of debt crisis on educational development in Latin America?

Ponencia presentada en "Annual Conference of the Comparative and International Education Society". Anaheim, Canadá.

Soto, F. (2000). Historia de la educación chilena. Santiago: CPEIP. 
Thompson, A. (2003). Caring in context: four feminist theories ongender and education. En: CurriculumInquiry, Vol. $33 \mathrm{~N}^{\circ} 1$, pp. 9-65.

Valdivieso, M. (1995). De madres a maestras. Tránsito de un saber. Santiago: Sernam.

Valcárcel, A. (2001). La memoria colectiva y los retos del feminismo. Santiago: CEPAL, Serie Mujer y Desarrollo, No 31. 